\title{
CONTRIBUIÇÃO AO ESTUDO DO BIMOMENTO EM ESTRUTURAS DE HASTES DE PAREDES DELGADAS
}

\author{
Vancler Ribeiro Alves ${ }^{1}$
}

Luiz Carlos Mendes ${ }^{2}$

\begin{abstract}
Resumo: O objetivo deste trabalho é avaliar a importância do comportamento de estruturas dotadas de elementos de hastes de paredes delgadas, e o surgimento das tensões de bimomentos nas mesmas. Particularizações do estudo são realizadas para uma viga monoengastada, dotada de solicitações que induzem ao surgimento de empenamentos e deformações características de hastes delgadas, utilizando-se os métodos analíticos. Para a análise mais abrangente do surgimento das tensões de bimomentos, cisalhantes e do comportamento estrutural são feitas variações nas dimensões da seção transversal dos perfis de seção aberta.
\end{abstract}

Palavras-chave: Estabilidade estrutural, bimomentos, hastes de paredes delgadas, análise estrutural.

Abstract: This work intends to evaluate bimoments stresses in thin-walled structures mainly in beams. External loads induce to warping and strains due to slenderness of thin profiles in open cross section, essential characteristics of a thin-walled structure. Analytical methods are employed in order to research the structural behavior and bimoments stresses, in a wider aspect that cannot be explained by the theory of thickwalled beams.

Keywords: Structural stability, bimoments, thin walled beams, structural analysis.

\section{INTRODUÇÃO}

A teoria das hastes de paredes delgadas foi desenvolvida pelo soviético V.S. Vlasov na segunda metade da década de trinta. Seu estudo baseou-se no efeito do empenamento elástico nas vigas de paredes delgadas através da teoria da flexo-torção, com a introdução de definições e análise das propriedades setoriais da seção, que em geral não são tratadas nos estudos iniciais da Mecânica Estrutural.

Entre as grandezas introduzidas por Vlasov encontram-se o bimomento e a torção de empenamento devida ao bimomento. Estas grandezas não podem ser desprezadas nos casos de perfis delgados submetidos à cargas axiais de

\footnotetext{
${ }^{1}$ Pós-Graduação em Engenharia Civil - UFF

${ }^{2}$ Pós-Graduação de Engenharia Civil - UFF
}

tração, compressão, momentos torçores e fletores, pois as tensões produzidas pelos bimomentos sobre a seção transversal dos perfis são significativas.

\section{FORMULAÇÃO DA TEORIA DO BIMOMENTO}

Seja o elemento infinitesimal de uma barra de haste delgada com comprimento $\mathrm{dx}$, submetida a cargas distribuídas $\mathrm{q}_{\mathrm{y}}, \mathrm{q}_{\mathrm{z}}$ e $\mathrm{m}_{\mathrm{t}}$, e aos esforços seccionais $N, V_{y}, V_{z}, M_{y}, M_{z}$ e $T$. As cargas $\mathrm{q}_{\mathrm{y}}$ e $\mathrm{q}_{\mathrm{z}}$ são uniformemente distribuídas ao longo das direções y e $\mathrm{z}$, respectivamente. A carga $\mathrm{m}_{\mathrm{t}}$ é $\mathrm{o}$ momento torçor uniformemente 
distribuído ao longo do eixo longitudinal

$\mathrm{x}$.

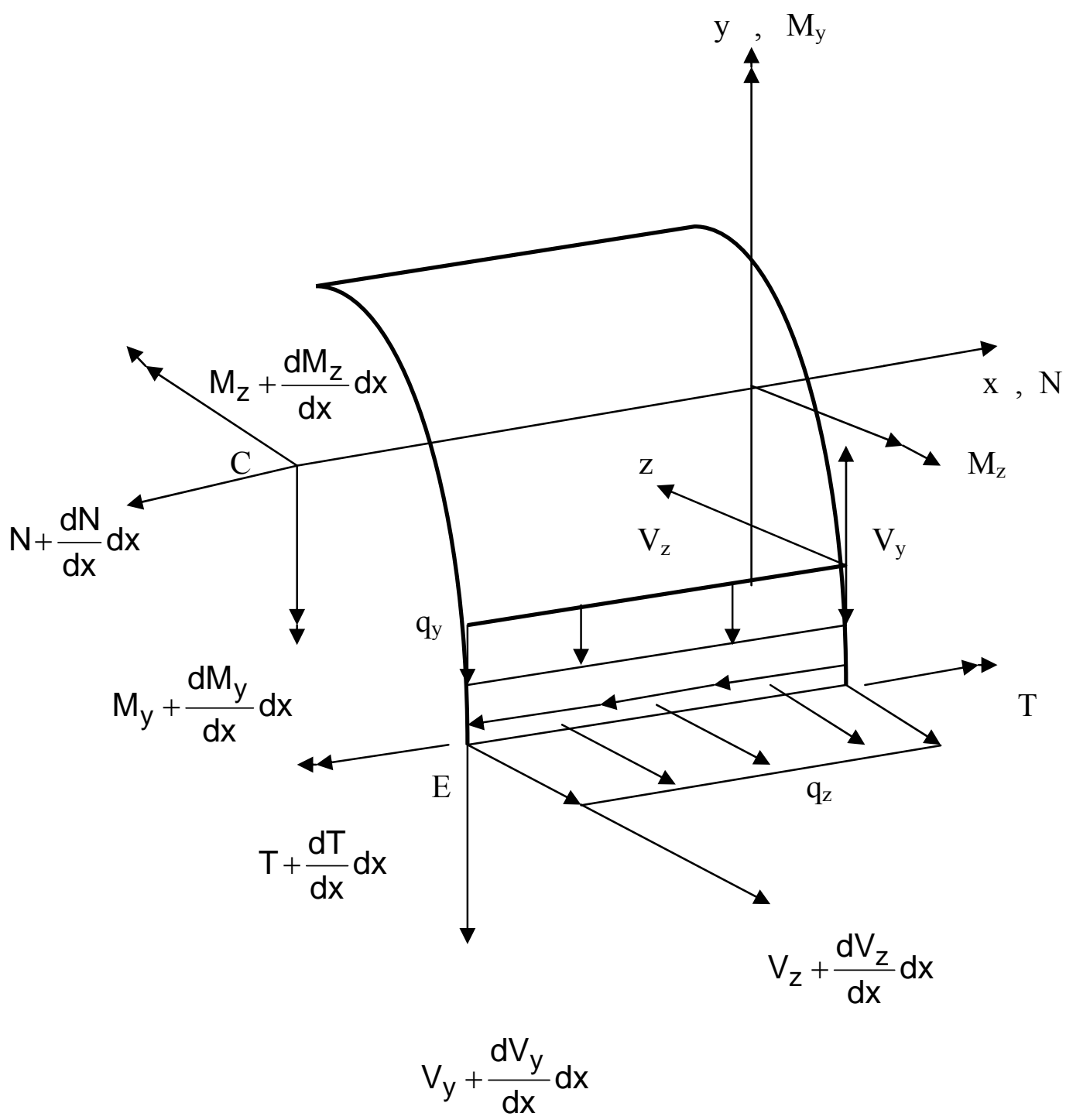

Figura 1. Cargas distribuídas e esforços seccionais atuantes em um elemento infinitesimal da barra.

$$
\frac{\mathrm{dN}}{\mathrm{dx}}=0
$$
$\mathrm{x}$ fornece:

O equilíbrio de forças na direção

$$
-N+N+\frac{d N}{d x} d x=0
$$

ou
Para o equilíbrio de forças na direção y tem-se:

$$
-V_{y}+q_{y} d x+V_{y}+\frac{d V_{y}}{d x} d x=0
$$

ou

$$
\frac{d V_{y}}{d x}=-q_{y}
$$

Obtém-se para o equilíbrio de forças na direção z: 


$$
\frac{\mathrm{dV}_{\mathrm{z}}}{\mathrm{dx}}=-\mathrm{q}_{\mathrm{z}}
$$

Sendo o eixo paralelo a $\mathrm{z}$ que passa pelo centróide da seção da direita, pode-se escrever para o equilíbrio de momentos:

$$
\begin{aligned}
& -M_{z}-V_{y} d x+\left(q_{y} d x\right) \frac{d x}{2}+M_{z}+ \\
& +\frac{d M_{z}}{d x} d x=0
\end{aligned}
$$

ou

$$
\frac{\mathrm{dM}_{\mathrm{z}}}{\mathrm{dx}}=\mathrm{V}_{\mathrm{y}}
$$

Por semelhança, para o eixo paralelo a y que passa pelo centróide da seção da direita, tem-se para o equilíbrio de momentos:

$$
\frac{\mathrm{dM}_{\mathrm{y}}}{\mathrm{dx}}=\mathrm{V}_{\mathrm{z}}
$$

Em relação ao eixo paralelo a $\mathrm{x}$ que passa pelo centro de cisalhamento, tem-se para o equilíbrio de momentos:

$$
-T+m_{t} d x+T+\frac{d T}{d x} d x=0
$$

ou

$$
\frac{\mathrm{dT}}{\mathrm{dx}}=-\mathrm{m}_{\mathrm{t}}
$$

A expressão para o esforço normal é representada por:

$$
\mathrm{N}=\mathrm{EA} \frac{\mathrm{du}_{0}}{\mathrm{dx}}-\mathrm{EA} \omega_{\mathrm{c}} \frac{\mathrm{d}^{2} \varphi}{\mathrm{dx}^{2}}
$$

onde:

$$
\mathrm{A}=\text { área da seção transversal; }
$$

$\mathrm{E}=$ módulo de elasticidade;

$\varphi=$ ângulo de rotação;

$\omega_{\mathrm{c}}=$ área setorial;

$\mathrm{u}_{0}=$ componente de deslocamento na direção x.

A substituição da equação (1) na equação (7), fornece:

$$
\frac{d^{2} u_{0}}{d x^{2}}-\omega_{c} \frac{d^{3} \varphi}{d x^{3}}=0
$$

A expressão para o momento torçor total é dada por:

$$
\begin{aligned}
& T=E A\left(\omega_{c}-\omega(L)\right) \frac{d^{2} u_{0}}{d x^{2}}- \\
& E\left(I_{\omega}-A \omega_{c} \cdot \omega(L)\right) \frac{d^{3} \varphi}{d x^{3}}+G K \frac{d \varphi}{d x}
\end{aligned}
$$

Substituindo-se a equação (6) na equação (9), e considerando-se a derivada em relação a x da equação (8), chega-se a:

$$
E\left(I_{\omega}-A \omega_{c}{ }^{2}\right) \frac{d^{4} \varphi}{d x^{4}}-G K \frac{d^{2} \varphi}{d x^{2}}=m_{t}
$$

Sendo a constante de flexo-torção representada por:

$$
\mathrm{C}_{\mathrm{w}}=\mathrm{I}_{\omega}-\mathrm{A} \omega_{\mathrm{c}}^{2}
$$

e, considerando-se a área setorial inicial nula, a constante de flexo-torção se resume ao momento setorial de inércia $I_{\omega}$.

Pode-se reescrever a equação (10) da seguinte maneira:

$$
\mathrm{EI}_{\omega} \frac{\mathrm{d}^{4} \varphi}{d \mathrm{x}^{4}}-\mathrm{GK} \frac{\mathrm{d}^{2} \varphi}{d \mathrm{x}^{2}}=\mathrm{m}_{\mathrm{t}}
$$

que fornece a função $\varphi(x)$, com a peça submetida à ação de um momento 
uniformemente distribuído de torção $\mathrm{m}_{\mathrm{t}}$ (x).

Obtém-se da equação (7) a seguinte relação:

$$
\frac{\mathrm{du}_{0}}{\mathrm{dx}}=\frac{\mathrm{N}}{\mathrm{EA}}+\omega_{\mathrm{c}} \frac{\mathrm{d}^{2} \varphi}{\mathrm{dx}^{2}}
$$

que fornece a equação da tensão $\sigma_{\mathrm{x}} \mathrm{da}$ seguinte maneira:

$$
\begin{gathered}
\sigma_{x}=\frac{N}{A}+\frac{M_{z} I_{y}-M_{y} I_{y z}}{I_{y} I_{z}-I_{y z}^{2}} y+ \\
+\frac{M_{y} I_{z}-M_{z} I_{y z}}{I_{y} I_{z}-I_{y z}^{2}} z-E\left(\omega-\omega_{c}\right) \cdot \frac{d^{2} \varphi}{d x^{2}}
\end{gathered}
$$

$$
\text { Para retirar-se o termo } \frac{d^{2} \varphi}{d x^{2}} d a
$$
equação (14), insere-se uma nova grandeza denominada bimomento, representada por $\mathrm{M}_{\omega}$.

O bimomento é definido pela expressão (15):

$$
M_{\omega}=\int \sigma_{x}\left(\omega-\omega_{c}\right) d A
$$

sendo a unidade de bimomento a força multiplicada pelo quadrado do comprimento. Substituindo-se a equação (14) na equação (15), e levando-se em consideração o fato do sistema de tensões ser auto-equilibrado, pode-se escrever:

$$
M_{\omega}=-E I_{\omega} \frac{d^{2} \varphi}{d x^{2}}
$$

sendo E. $\mathrm{I}_{\omega}$ denominada rigidez ao empenamento, sendo $I_{\omega}$ definido como momento setorial de inércia da haste, expresso de acordo com o tipo de seção transversal.

\section{EQUAÇÃO DIFERENCIAL DO BIMOMENTO}

Derivando-se duas vezes a equação (16) em relação a $x$, pode-se escrever:

$$
\frac{d^{4} \varphi}{d x^{4}}=-\frac{1}{E I_{\omega}} \frac{d^{2} M_{\omega}}{d x^{2}}
$$

A equação diferencial do bimomento é obtida com a substituição das equações (17) e (16) na equação (12):

$$
\frac{\mathrm{d}^{2} \mathrm{M}_{\omega}}{\mathrm{dx}^{2}}-\frac{\mathrm{GK}}{\mathrm{EI_{ \omega }}} \mathrm{M}_{\omega}=-\mathrm{m}_{\mathrm{t}}
$$

\section{ANÁLISE DE VIGA SOB EMPENAMENTO}

Com o auxílio da computação algébrica simbólica (CAS - MathCad 2001), será feita a análise dos bimomentos para uma viga monoengastada, de comprimento $\mathrm{L}=10$ $\mathrm{m}$, sujeita a um carregamento torsional uniformemente distribuído $\mathrm{m}_{\mathrm{D}}=1$ $\mathrm{kNm} / \mathrm{m}$, para um grupo de perfis viga soldada. Os perfis são considerados hastes de paredes delgadas pelo critério de Zbirohowski-Koscia (1967), onde o comprimento característico inverso $\mathrm{k}$ é menor que $1,27 \mathrm{~cm}^{-1}$. O grupo foi subdividido em quatro partes, compostos de seis perfis para facilidade de análise em gráficos do MathCad. A viga é discretizada em 11 pontos distintos, a partir do engaste, distantes $1 \mathrm{~m}$ entre si, sendo os valores dos bimomentos expressos por Kollbrunner (1972, p.129):

$$
\begin{aligned}
& M_{\omega}=\frac{1}{k^{2}}[1+(k L \operatorname{senh}(k x)- \\
& \left.-\frac{1+k L \operatorname{senh}(k L)}{\cosh (k L)} \cosh (k x)\right]
\end{aligned}
$$




$$
\mathrm{m}_{\mathrm{D}}=1 \mathrm{kNm} / \mathrm{m}
$$

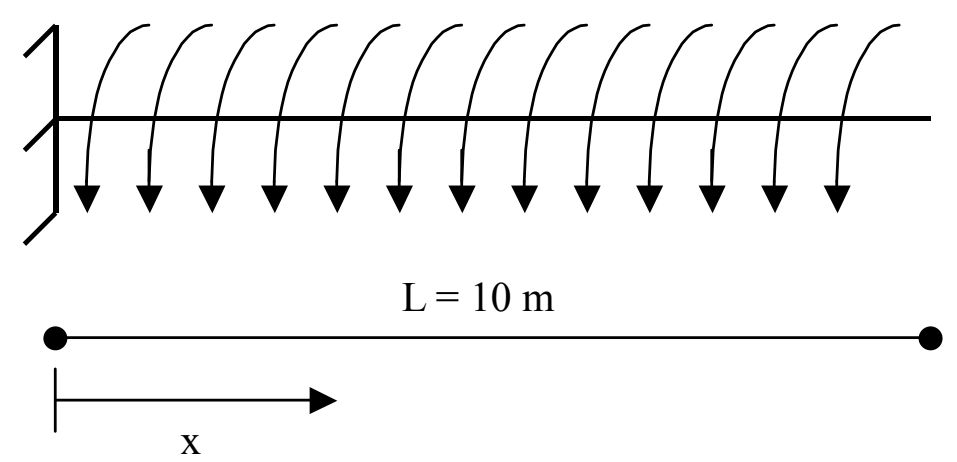

Figura 2. Haste monoengastada sujeita a carregamento torsional uniformemente distribuído.

A subdivisão dos agrupamentos está indicada abaixo.

Tabela 1. Agrupamentos dos perfis em análise.

\begin{tabular}{|c|c|c|c|}
\hline $1^{\circ}$ GRUPO & $2^{\circ}$ GRUPO & $3^{\circ}$ GRUPO & $4^{\circ}$ GRUPO \\
\hline VS $250 \times 28$ & VS $400 \times 49$ & VS $700 \times 105$ & VS $1000 \times 140$ \\
\hline VS 275 X 30 & VS $450 \times 51$ & VS $750 \times 108$ & VS $1100 \times 159$ \\
\hline VS $300 \times 33$ & VS $500 \times 61$ & VS $800 \times 111$ & VS $1200 \times 200$ \\
\hline VS $325 \times 35$ & VS $550 \times 64$ & VS $850 \times 120$ & VS $1300 \times 237$ \\
\hline VS $350 \times 38$ & VS $600 \times 95$ & VS $900 \times 124$ & VS $1400 \times 260$ \\
\hline VS 375 X 40 & VS $650 \times 98$ & VS $950 \times 127$ & VS $1500 \times 270$ \\
\hline
\end{tabular}

A análise procede com a apresentação dos seis gráficos com os valores de bimomentos em kN.cm no eixo vertical em função dos pontos da haste no eixo horizontal. 


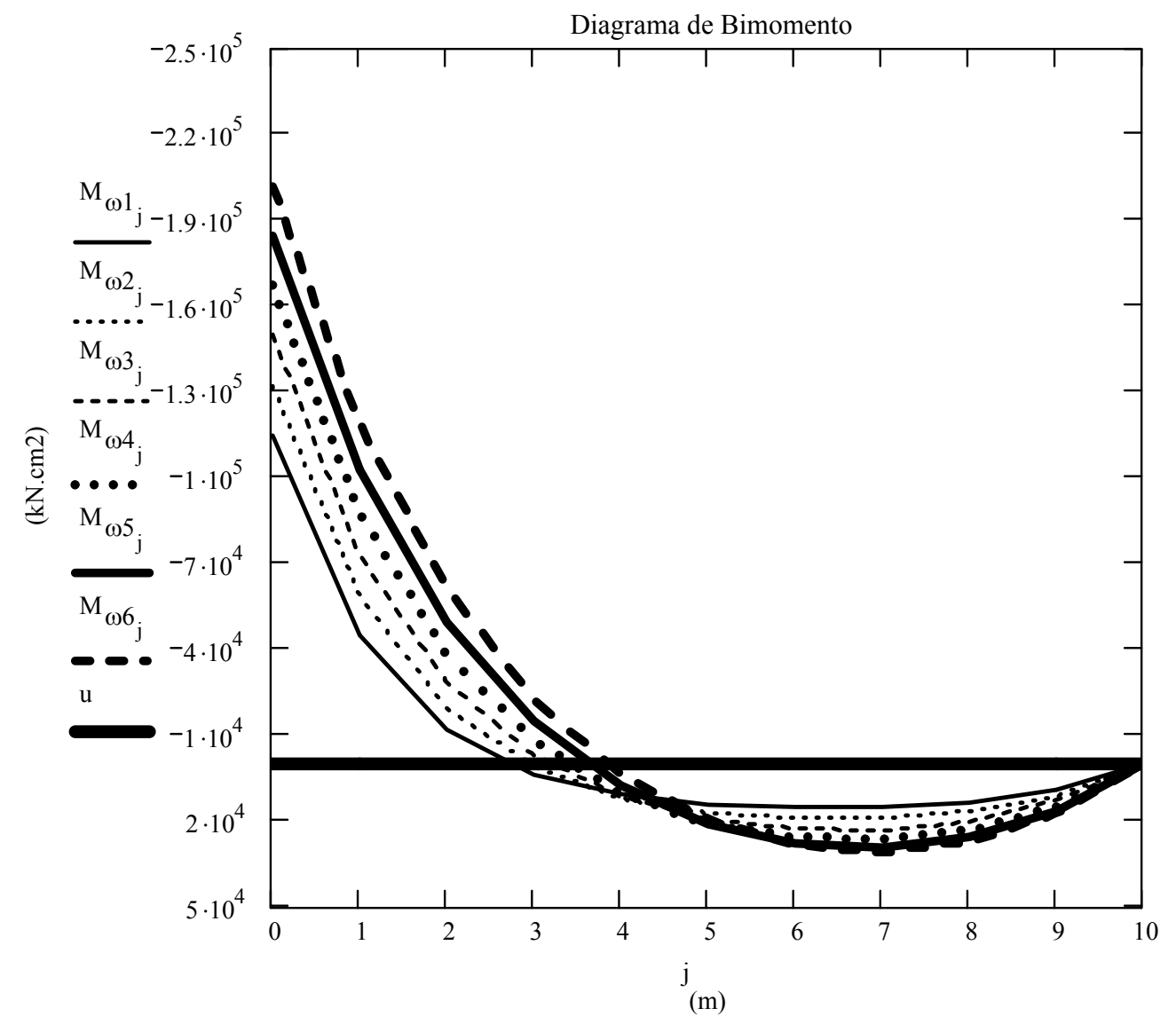

Figura 3. Bimomentos em kN.cm² na haste para os perfis do primeiro grupo.

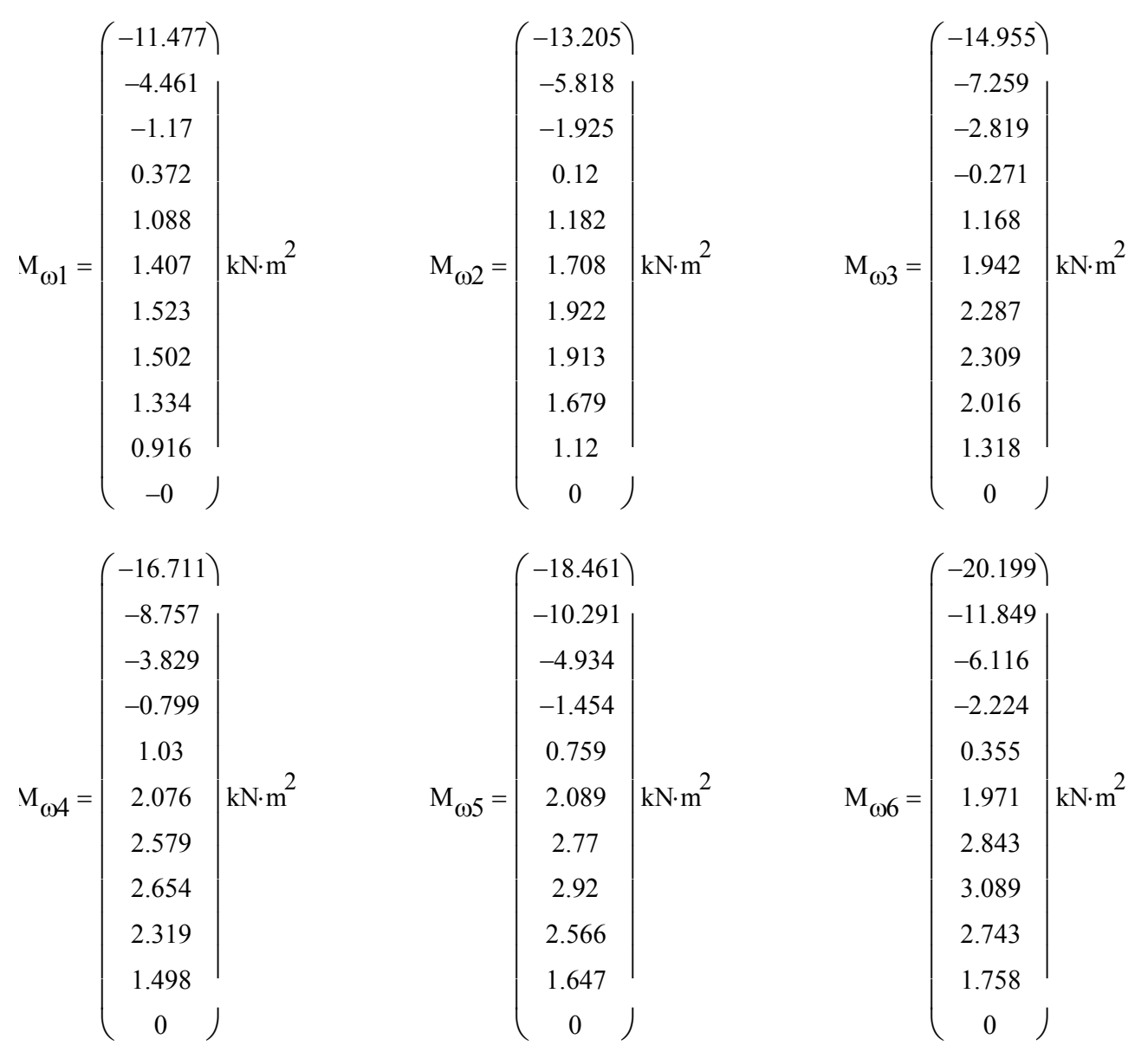




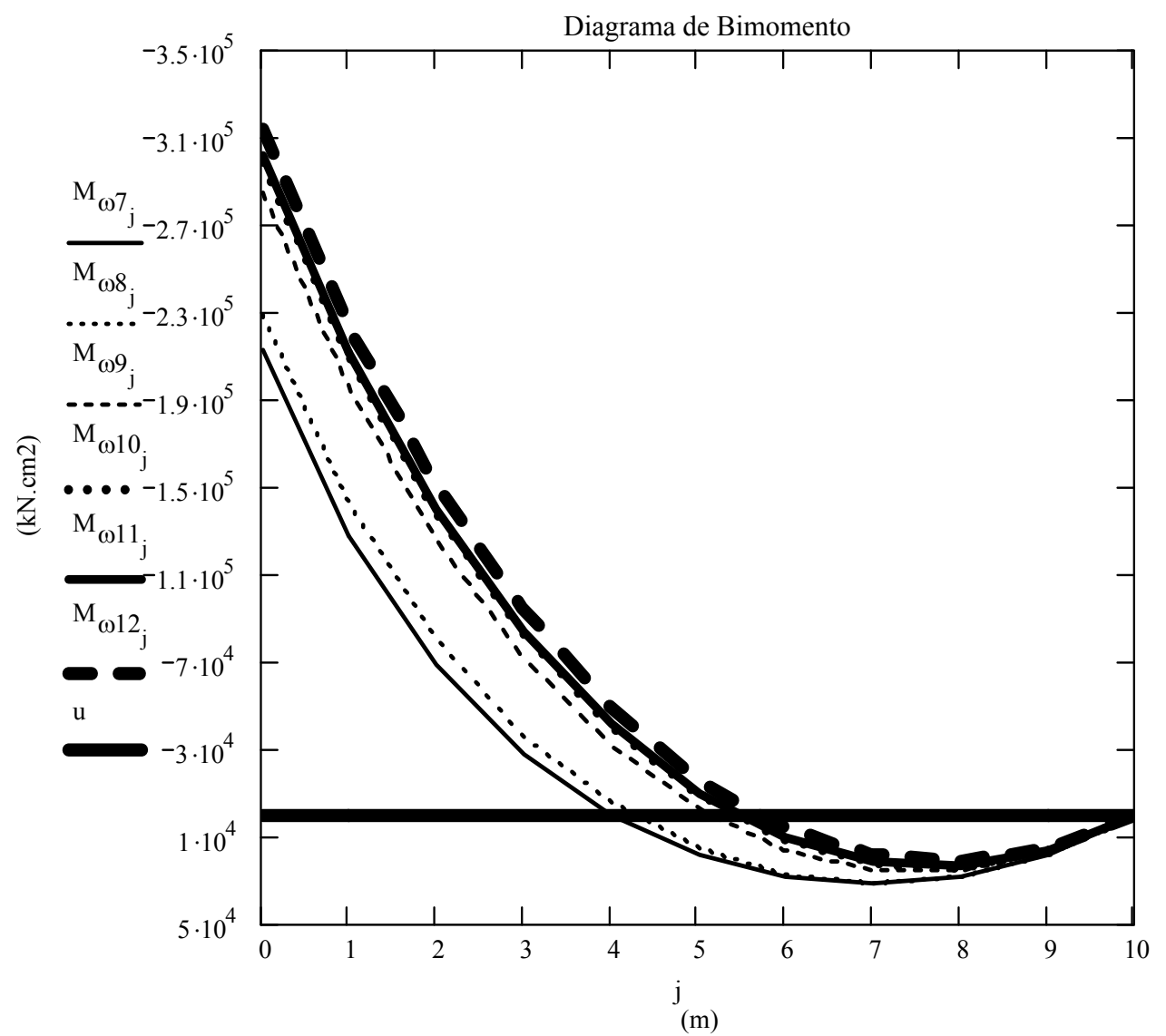

Figura 4. Bimomentos em $\mathrm{kN} . \mathrm{cm}^{2}$ na haste para os perfis do segundo grupo.

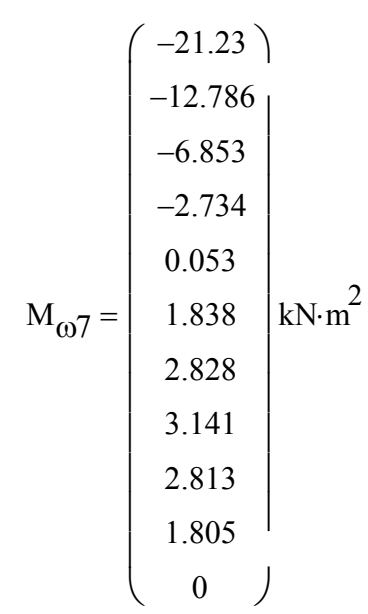

$\mathrm{M}_{\omega 10}=\left(\begin{array}{c}-29.885 \\ -20.917 \\ -13.789 \\ -8.214 \\ -3.971 \\ -0.89 \\ 1.153 \\ 2.238 \\ 2.409 \\ 1.673 \\ 0\end{array}\right) \mathrm{kN} \cdot \mathrm{m}^{2}$
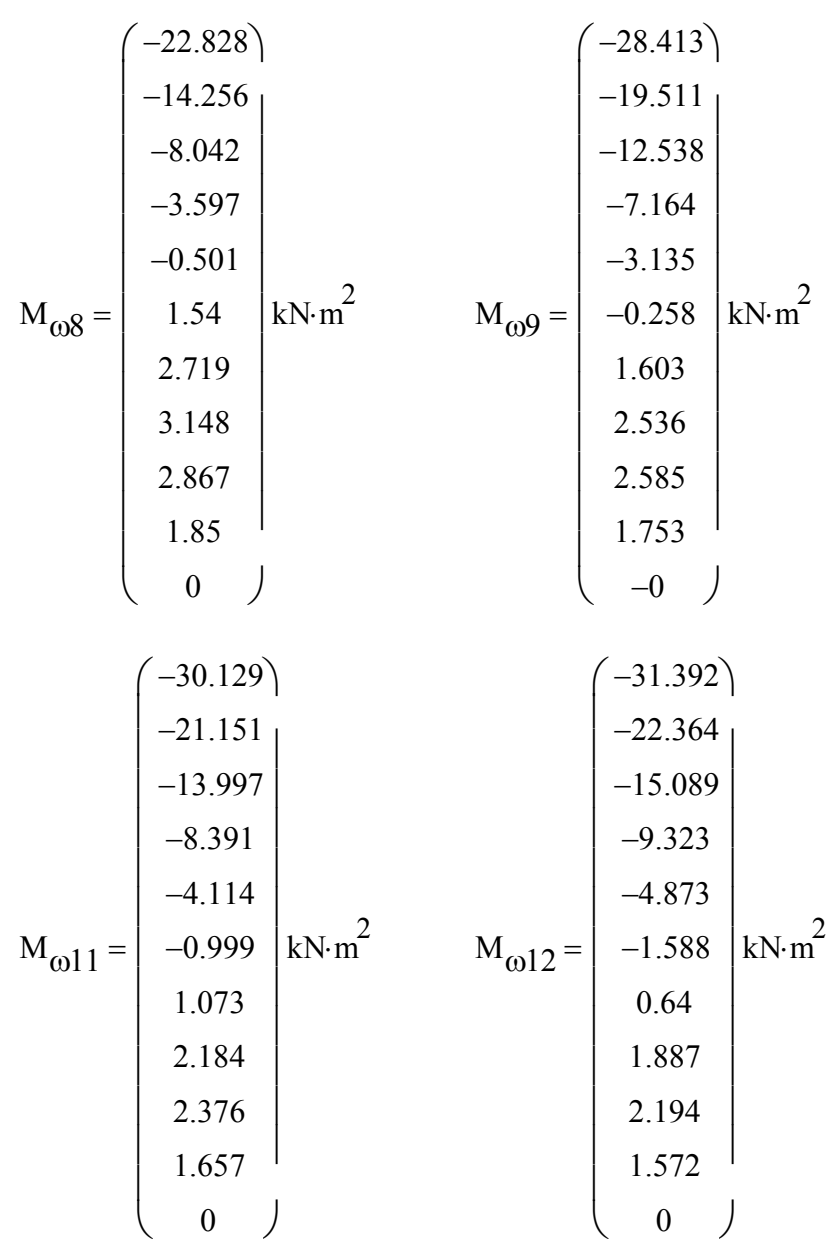


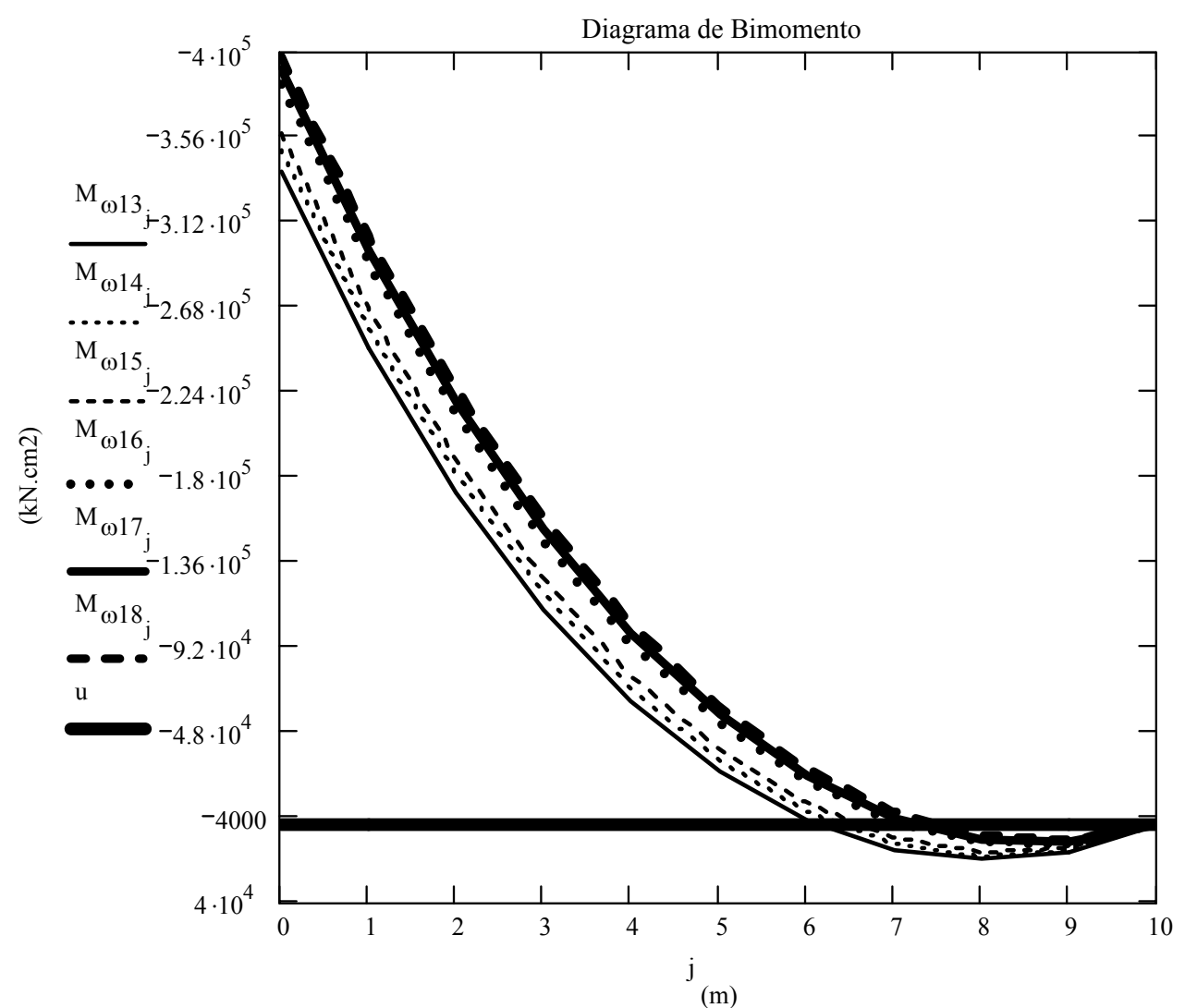

Figura 5. Bimomentos em kN.cm² na haste para os perfis do terceiro grupo.

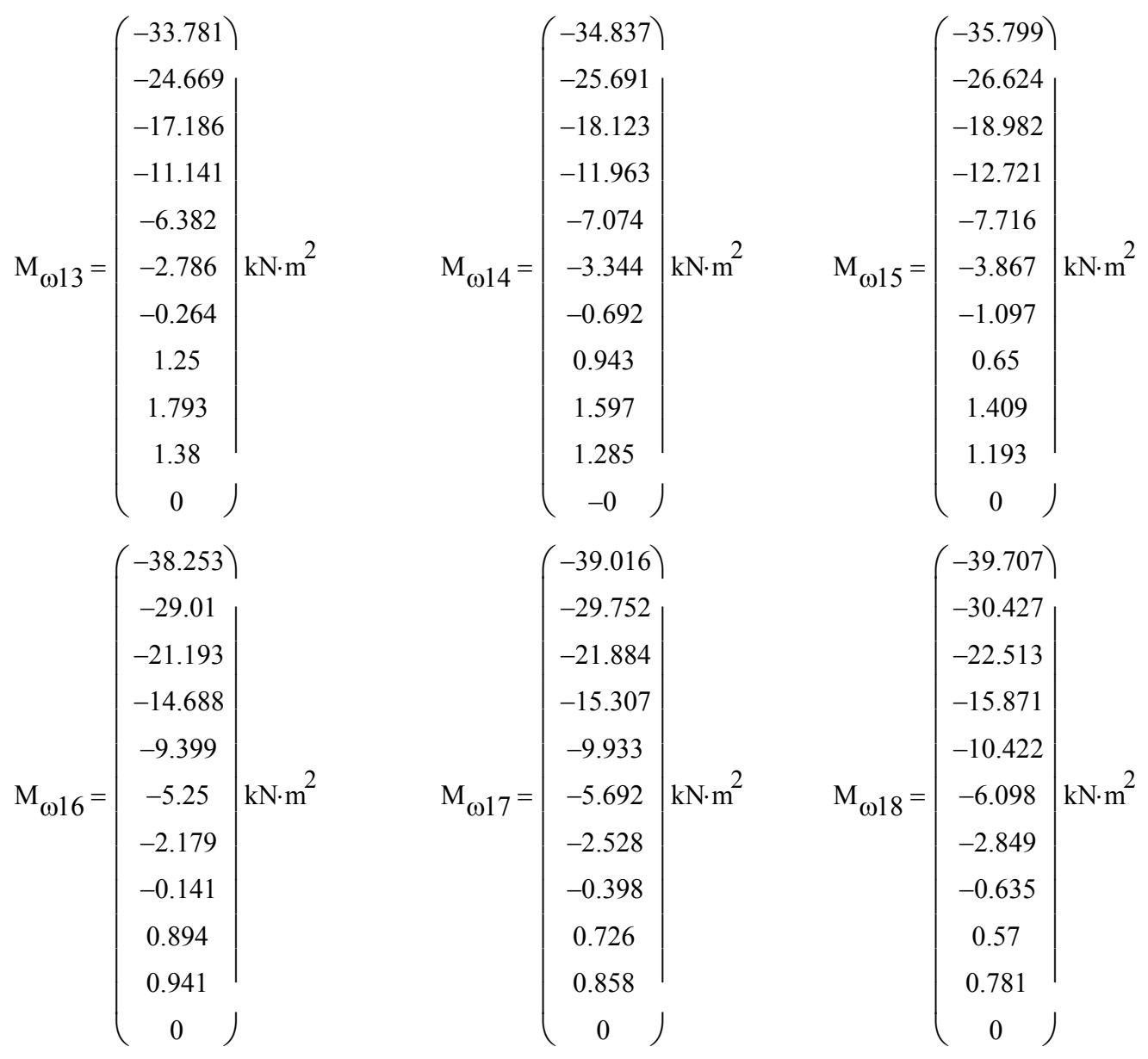




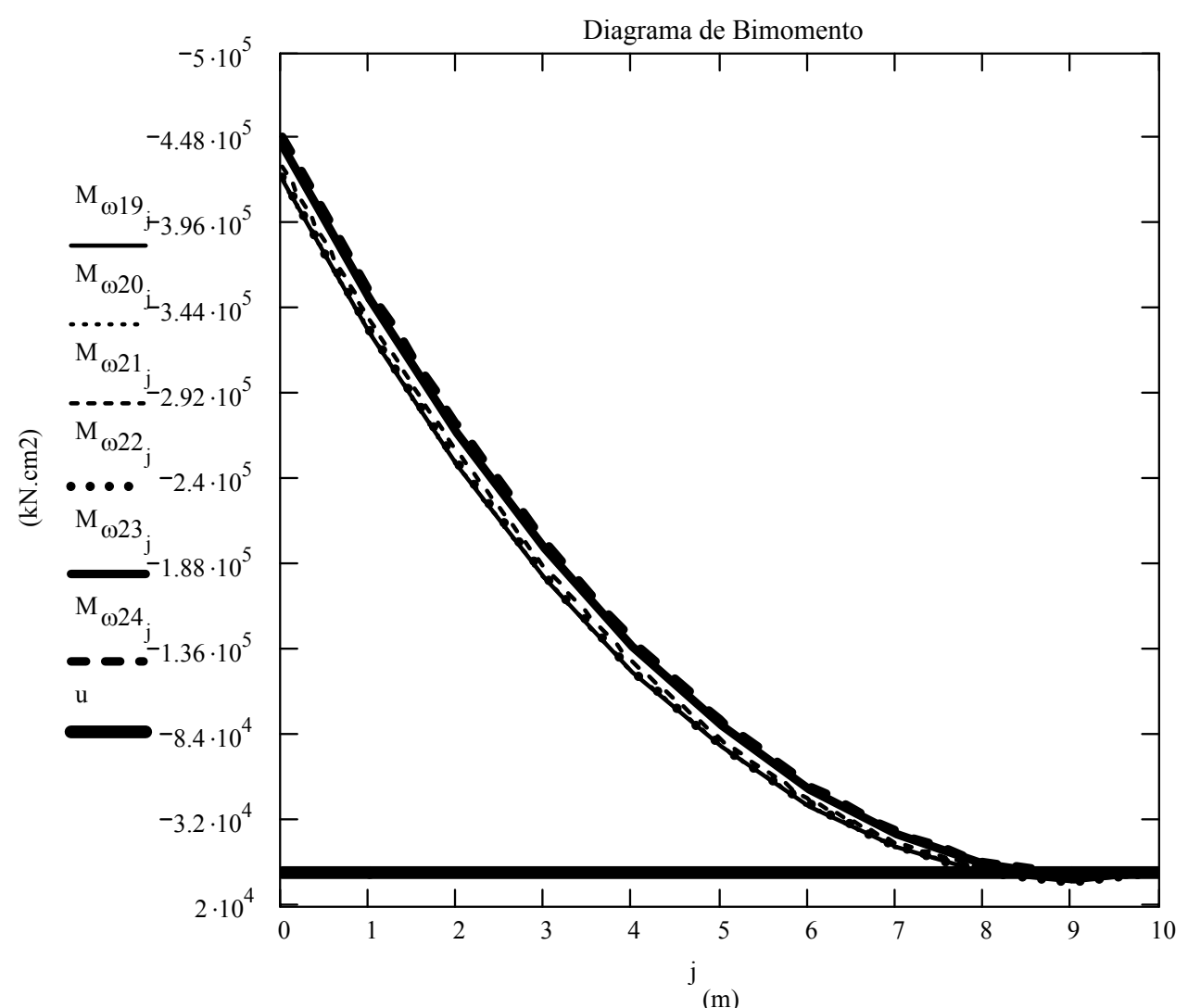

Figura 6. Bimomentos em kN.cm² na haste para os perfis do quarto grupo.

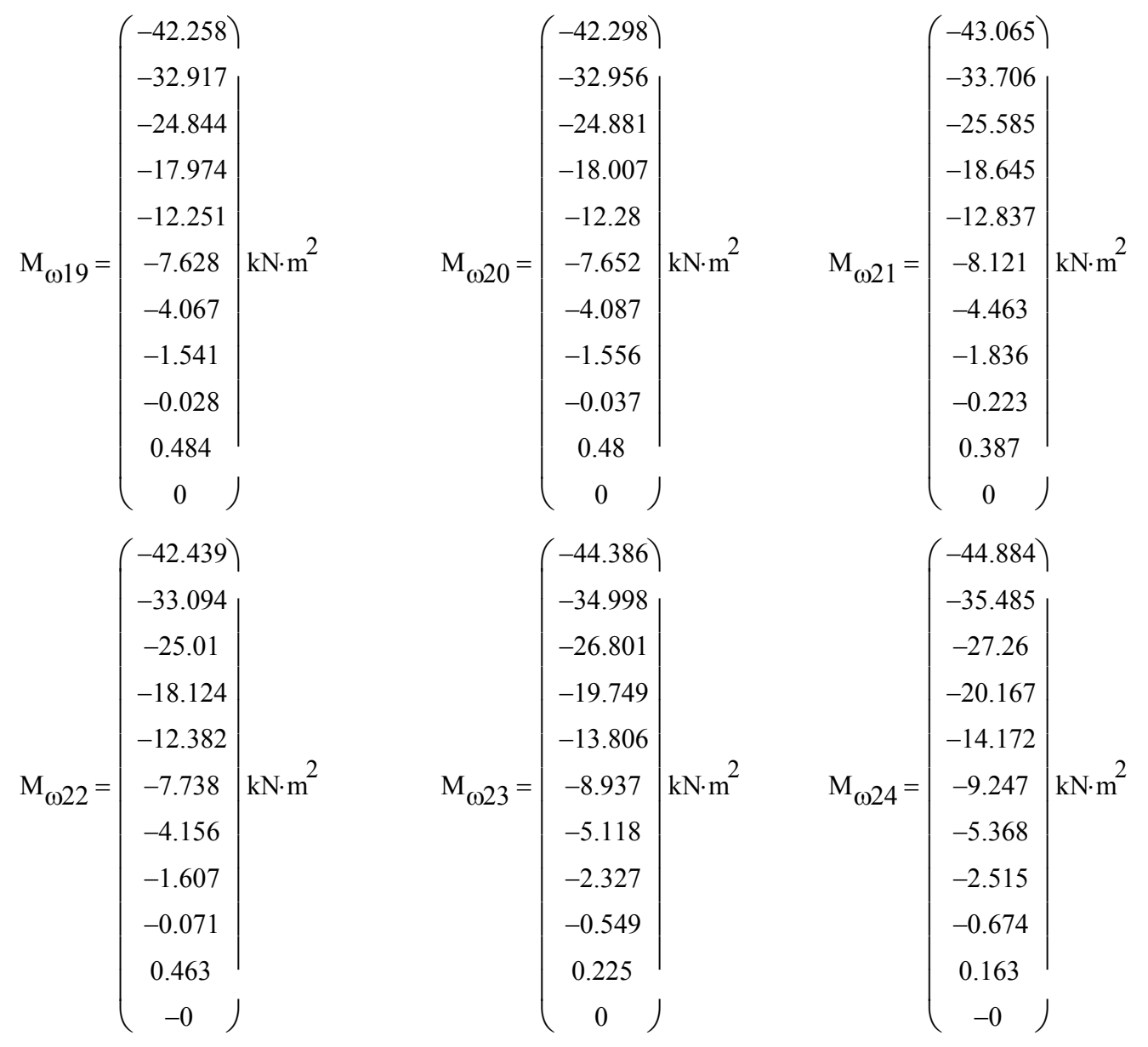




\section{DISCUSSÃO DOS RESULTADOS}

Para os perfis do primeiro grupo, existe um ponto de valor comum para o bimomento positivo localizado próximo da metade do vão da haste. Valores máximos positivos ocorrem no terço próximo ao bordo livre da haste. Os perfis mais delgados, aqueles de menor comprimento característico inverso $\mathrm{k}$, apresentam os maiores valores de bimomentos positivos e negativos.

Nos perfis do segundo grupo, há acréscimo de bimomento negativo no engaste em comparação aos perfis do grupo anterior. Os perfis mais delgados apresentam maiores valores de bimomentos negativos, e menores valores de bimomentos positivos.

Para os perfis do terceiro grupo, há acréscimo de bimomento negativo no engaste, e decréscimo de bimomento positivo em comparação aos perfis do grupo anterior.

Nos perfis do quarto grupo, ocorrem pequenas variações. Há acréscimo de bimomento negativo no engaste em relação aos perfis do grupo anterior. Os bimomentos são reduzidos a partir do segundo trecho próximo ao bordo livre da haste, generalizadamente.

\section{CONCLUSÕES}

A importância do empenamento da seção transversal fica clara quando se aumentam todas as dimensões da seção transversal paralelamente com a altura do perfil, em uma haste monoengastada sujeita a um carregamento torsional. Quanto mais os perfis vão se tornando delgados, indicados pela diminuição do valor do coeficiente $\mathrm{k}$, maiores são os valores dos bimomentos despertados na extremidade engastada, e menores são as tensões normais devidas ao bimomento na mesma extremidade engastada. Isto se deve ao fato de os momentos setoriais de inércia experimentarem um acréscimo exagerado, numa taxa bem superior a dos bimomentos e a do coeficiente de empenamento $\omega$.

\begin{abstract}
Observa-se que nem sempre os maiores bimomentos positivos são daqueles perfis que apresentam os maiores bimomentos negativos, ocorrendo uma verdadeira inversão nos seus diagramas dentro de um mesmo agrupamento. Tal inversão de sinais se verifica naqueles perfis de maior comprimento característico inverso e a redução dos valores em módulo dos bimomentos ocorre em trechos próximos ao bordo livre da haste de uma forma geral.
\end{abstract}

\section{BIBLIOGRAFIA}

ALVES, Vancler Ribeiro. Análise de elementos estruturais em hastes de paredes delgadas. Tese de Mestrado, UFF, Niterói, 2003.

ALVES, Vancler Ribeiro; MENDES, Luiz Carlos. Métodos matriciais em estruturas de hastes de paredes delgadas. ENGEVISTA, v.5, n8, p. 53-65, Niterói, 2003.

DOS SANTOS, Luciano Barbosa; MORI, Dalgoberto Dario; DE SALES José Jairo. Influência do bimomento no comportamento de perfis $\mathrm{Z}$ submetidos à força axial centrada de tração. XXX Jornadas Sul-Americanas de Engenharia Estrutural, CD-ROM, Brasília, 2002.

CHEUNG, Y. K.; KOO, K. K. Analytical method for thin-walled members in general bending and torsion. Thin-Walled Structures, Elsevier Science Ltd., Exeter, England, v. 6, n. 5, p. 355-369, 1988.

CHILVER, A. H. Thin-walled structures, John Wiley \& Sons Inc., New York, 1974.

ILG, I. Vigas contínuas em hastes de paredes delgadas, Tese de Mestrado, COPPE/UFRJ, Rio de Janeiro, 1983.

JÖNSSON, Jeppe. Determination of shear stresses, warping functions and sections properties of thin-walled beams using finite elements. Computers \& $\underline{\text { Structures }}$, Elsevier Science Ltd., Exeter, England, v. 68, n. 6, p. 393-410, 1998. 
KOLLBRUNNER, C. F.; HAJDIN, N. Dünnwandige Stäbe - Band 1, Berlin, Springer Verlag, 1972.

KOLLBRUNNER, C. F.; BASLER, K. Torsion in Structures - An Engineering Approach, Springer - Verlag, Berlin/Heidelberg/New York, 1969.

KOLLBRUNNER, C. F.; HAJDIN, N.; KAJCINOVIC, D. Matrix analysis of thin-walled structures, Institute for Engineering Research, Verlag / Leemann / Zurich, 1969.

LEITÃO, M. A. S. Hastes curtas de paredes delgadas, Tese de Doutorado, COPPE/UFRJ, Rio de Janeiro, 1991.

MENDES, Luiz Carlos. Análise de grelhas em hastes de paredes delgadas sujeitas a bimomentos, Tese de Doutorado, COPPE/UFRJ, Rio de Janeiro, 1993.

MENDES, Luiz Carlos. Contribuição ao estudo das hastes de paredes delgadas, Tese para Professor Titular, UFF, Niterói, 1993.

MURRAY, N. W. Introduction to the theory of thin-walled structures, Oxford, Engineering Science v. 13, 1986.

ODEN, J. T. Mechanics of elastic structures, New York, McGraw-Hill Book Company, 1967.

PAPANGELIS, J. P.; HANCOCK, G. J. Computer analysis of thin-walled structural members. Computers \& Structures, Elsevier Science Ltd., Exeter, England, v. 56, n. 1, p. 157-176, 1995.

PERES, S. V. S.; BATISTA, E. M. Torção não uniforme em perfis de paredes finas de seção aberta: estudo teórico-experimental. XXX Jornadas SulAmericanas de Engenharia Estrutural, CD-ROM, Brasília, 2002.

POLILLO, V. R.; GARCIA, L. F. T.; VILLAÇA, S. F. Tensões oriundas da flexo-torção em hastes delgadas de seção aberta. Revista Brasileira de Engenharia - Caderno de Engenharia Estrutural, ABPE, Rio de Janeiro, v. 8, n. 1, p. 3757, 1991.
PROKIC, A. Thin-walled beams with open and closed cross-sections. Computers and Structures, Elsevier Science Ltd., Exeter, England, v. 47, n. 6, p. 1065-1070, 1993.

RHODES, J. Design of cold formed steel members, Elsevier Science Publishers Ltd., London, 1991.

SÁNCHEZ FILHO, Emil de Souza. Torção de peças de seção aberta com hastes de paredes delgadas de concreto armado, Tese de Mestrado, COPPE/UFRJ, Rio de Janeiro, 1988.

SHAMES, I. H.; DYM, C. L. Energy and finite element methods in structural mechanics, McGraw-Hill Book Company Inc., New York, 1985.

ZBIROHOWSKI-KOSCIA, K. Thin walled beams, Crosby Lockwood \& Son Ltd., London, 1967. 